\title{
Extrapolation of In Vivo Hepatic Clearance from In Vitro Uptake Clearance by Suspended Human Hepatocytes for Anionic Drugs with High Binding to Human Albumin: Improvement of In Vitro-to-In Vivo Extrapolation by Considering the "Albumin-Mediated" Hepatic Uptake Mechanism on the Basis of the "Facilitated-Dissociation Model" [\$
}

\author{
Soo-Jin Kim, ${ }^{1,2}$ Kyeong-Ryoon Lee, ${ }^{1,3}$ Seiji Miyauchi, and Yuichi Sugiyama \\ Sugiyama Laboratory, RIKEN Baton Zone Program, RIKEN Cluster for Science, Technology and Innovation Hub, RIKEN, \\ Tsurumi-ku, Yokohama, Japan (S.-J.K., K.-R.L., Y.S.); Life Science Institute, Daewoong Pharmaceutical, Pogok-eup, Cheoin-gu, \\ Yongin, Korea (K.-R.L.); and Faculty of Pharmaceutical Sciences, Toho University, Funabashi, Chiba, Japan (S.M.)
}

Received July 31, 2018; accepted November 27, 2018

\begin{abstract}
We investigated whether human serum albumin (HSA) in suspended human hepatocytes would affect the uptake clearance of anionic drugs with high binding to HSA and improve the extrapolation of in vivo hepatic clearance from in vitro uptake clearance by the hepatocytes via the "albumin-mediated" hepatic uptake mechanism. The uptake clearances for total forms $\left(P S_{\text {inf }}\right)$ and for unbound forms $\left(P S_{\mathrm{u}, \mathrm{inf}}\right)$ of 11 anionic drugs [all of which were organic aniontransporting polypeptide (OATP) substrates] were determined with suspended human hepatocytes in varying concentrations of HSA. The fraction of unbound drugs $\left(f_{\mathrm{u}}\right)$ was determined using an equilibrium dialysis at the various HSA concentrations. The $P S_{\text {inf }}$ values decreased with increasing concentrations of HSA, whereas the unbound uptake clearances $\left(P S_{\mathrm{u}, \mathrm{inf}}(+)=P S_{\mathrm{inf}} / f_{\mathrm{u}}\right)$ in the presence
\end{abstract}

of HSA increased substantially, thus demonstrating the "albuminmediated" hepatic uptake mechanism. The relationships between $P S_{\text {inf }}$ and HSA concentration were well described by the previously proposed facilitated-dissociation model, in which the drug-albumin complex interacts with the cell surface, enhancing the dissociation of the complex and providing unbound drug for hepatic uptake. Furthermore, the $P S_{u, \text { inf }}(+)$ values in in vivo conditions (at $5 \%$ HSA) were predicted from those obtained in isolated hepatocytes on the basis of the facilitated-dissociation model, revealing compatibility with the overall hepatic intrinsic clearance in vivo. We conclude that the "facilitated-dissociation" model is useful for describing the "albumin-mediated" hepatic uptake phenomenon of OATP drugs and to predict hepatic uptake clearance in vivo.

\section{Introduction}

It is very important that the pharmacokinetic features of new chemical entities in humans be adequately predicted during early stages of drug discovery and development. The prediction of human hepatic clearance is crucial because the liver is the major organ responsible for elimination of a variety of endogenous and exogenous

This study was supported by Grant-in-Aid for Scientific Research (C) and (S) from the Japanese Ministry of Education, Culture, Sports, Sciences, and Technology [Grant 26460044] and [Grant 24229002], respectively.

${ }^{1}$ S.-J.K. and K.-R.L. contributed equally to this work.

${ }^{2}$ Current affiliation: Drug Evaluation Center, R\&D Institute, CJ HealthCare, Majang-myeon, Icheon, Korea.

${ }^{3}$ Current affiliation: Laboratory Animal Resource Center, Korea Research Institute of Bioscience and Biotechnology, Cheongwon-gu, Cheongju, Korea.

https://doi.org/10.1124/dmd.118.083733.

SThis article has supplemental material available at dmd.aspetjournals.org. chemicals via metabolism and/or biliary excretion. Currently, humanderived reagents, such as human hepatic microsomes, cytosols, and suspended hepatocytes, are available for in vitro studies, greatly improving the quantitative in vitro-to-in vivo extrapolation (IVIVE) for hepatic clearance values (Watanabe et al., 2011; Izumi et al., 2017). Compared with anion drugs, anionic drugs with high plasma binding tend to have poor predictive accuracy for human hepatic clearances, because they require mechanistic elucidation and quantitative improvement (Watanabe et al., 2011; Izumi et al., 2017).

Poulin et al. (2016) retrieved the in vitro and in vivo hepatic clearances of several drugs with high albumin binding and found that the conventional IVIVE procedure whose basis is the "free drug" hypothesis largely underestimated the in vivo hepatic clearances. Poulin et al. proposed an adjustment procedure to obtain the unbound drug concentration under in vivo conditions by considering differences in $\mathrm{pH}$ between intracellular and extracellular spaces and in albumin concentrations between the plasma and liver, as well as

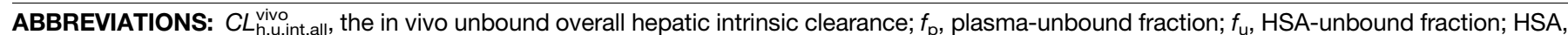
human serum albumin; IVIVE, in vitro-to-in vivo extrapolation; OATP, organic anion transporting polypeptide; $P S_{\mathrm{b}, \text { inf }}$, the hepatic uptake clearance for the unbound drug dissociated from the drug-albumin complex at the surface; $P S_{\text {inf, }}$ the hepatic uptake clearance for total drug; $P S_{\mathrm{u} \text {,inf }}$, the hepatic uptake clearance for unbound drug; $P S_{\mathrm{u}, \text { inf }}(+)$, the hepatic unbound uptake clearance in the presence of the albumin; PRV, pravastatin; $R$, hepatic uptake clearance; RSV, rosuvastatin; $S F$, scaling factor. 
pH-partition mechanisms. This adjustment procedure improved the predictive accuracy of hepatic clearances for drugs that bind avidly to albumin.

We recently demonstrated that organic anion-transporting polypeptide (Oatp/OATP) substrates were taken up by primary cultured rat hepatocytes, and human suspended hepatocytes in the presence of albumin, to a much greater extent than could be expected on the basis of their unbound concentration and the "free drug" hypothesis (Miyauchi et al., 2018). The enhancement of the hepatic uptake (often referred to as "albumin-mediated" hepatic uptake) has been described in the "facilitated-dissociation" model, in which the drug-albumin complex interacts with the cell surface, enhancing the dissociation of the complex and providing unbound drug for hepatic uptake (Tsao et al., 1988a). Considering the "albumin-mediated" hepatic uptake, IVIVE for hepatic uptake clearance improves prediction accuracy. Using an isolated perfused rat liver system, Bounakta et al. (2018) and Poulin et al. (2017) also demonstrated that the IVIVE method considering the "albumin-mediated" hepatic uptake mechanism improved the predictive accuracy of in vivo hepatic clearance compared with their empirical IVIVE that considered $\mathrm{pH}$ and albumin concentration differences between the plasma and liver. Together, these results provide solid evidence supporting a kinetic model for describing the "albumin-mediated" hepatic uptake phenomenon for a drug avidly bound to albumin, which is important for robust IVIVE.

In the present study, our first objective was to investigate the effect of human serum albumin (HSA) on the uptake clearances of 11 drugs known as OATP substrates using suspended human hepatocytes. Our second objective was to improve the prediction of quantitative IVIVE for hepatic uptake clearance values by considering "albuminmediated" hepatic uptake mechanisms on the basis of the "facilitateddissociation" model.

\section{Materials and Methods}

Materials. Pitavastatin calcium salt, atorvastatin calcium trihydrate, rosuvastatin (RSV) calcium salt, fluvastatin sodium salt, and pravastatin (PRV) sodium salt were purchased from Wako Pure Chemicals (Kyoto, Japan). Cerivastatin sodium salt and repaglinide were purchased from LKT Laboratories (St. Paul, MN). Glibenclamide (GLB), nateglinide, and valsartan were purchased from Tokyo Chemical Industry Co., Ltd. (Tokyo, Japan). Bosentan was purchased from Toronto Research Chemicals Inc. (North York, ON, Canada). Human plasma and HSA (fatty acid free) were purchased from MilliporeSigma (St. Louis, MO). All other reagents used were of analytical or high-performance liquid chromatography grade.

Unbound Fraction in Human Plasma and HSA. Plasma protein binding of 11 compounds was determined in $100 \%$ human plasma and six different concentrations $(0 \%, 0.125 \%, 0.25 \%, 0.5 \%, 1 \%$, and $5 \%$ or $0,18.8,37.6,75.2$, 150 , and $752 \mu \mathrm{M}$ ) of HSA by equilibrium dialysis with a Rapid Equilibrium Dialysis (RED) Device (Thermo Fisher Scientific, Rockford, IL). Various concentrations of HSA were prepared with transporter uptake buffer ( $\mathrm{pH}$ 7.4). The final concentration of compounds in human plasma or HSA samples was $3 \mu \mathrm{M}$. Aliquots $(100 \mu \mathrm{l})$ of each sample and phosphate-buffered saline or transporter uptake buffer $(300 \mu \mathrm{l})$ were added to the equilibrium dialysis device. The plate was put into a $37^{\circ} \mathrm{C}$ incubator and shaken gently for 20 hours. Aliquots of samples $(25-\mu \mathrm{l})$ and $50-\mu \mathrm{l}$ aliquots of buffers were then taken and mixed with acetonitrile containing an internal standard. The mixture was vigorously mixed and centrifuged at $4^{\circ} \mathrm{C}$. An aliquot $(5 \mu \mathrm{l})$ of each supernatant was injected into a liquid chromatography-tandem mass spectrometry (LC-MS/MS) system (Supplemental Table S1).

Uptake Studies in Suspended Human Hepatocytes. The uptake rate of all compounds was determined using pooled cryopreserved human hepatocytes (Lot TFF, 20 mixed-gender donors) (BioIVT, Baltimore, MD) by a centrifugal filtration technique described previously (Hirano et al., 2004). Immediately prior to the uptake experiment, the cryopreserved hepatocytes $(1 \mathrm{ml}$ suspension) were thawed at $37^{\circ} \mathrm{C}$, then suspended in $50 \mathrm{ml}$ of prewarmed cryopreserved hepatocyte recovery media thawing medium (APSciences, Inc., Columbia, MD) and centrifuged $(100 \mathrm{~g})$ for 2 minutes at $4{ }^{\circ} \mathrm{C}$, followed by removal of the supernatants. The hepatocytes were gently resuspended in icecold Krebs-Henseleit buffer to give $2.0 \times 10^{6}$ viable cells $/ \mathrm{ml}$ on ice. The uptake studies were initiated by adding an equal volume of prewarmed buffer containing two sets of drugs: 1) pitavastatin, atorvastatin, RSV, fluvastatin, cerivastatin, and PRV; 2) glibenclamide, valsartan, repaglinide, bosentan, and nateglinide, with six different concentrations of HSA $(0 \%, 0.125 \%, 0.25 \%$, $0.5 \%, 1 \%$, and $5 \%$ ), added to the cell suspension after a 5 -minute preincubation at $37^{\circ} \mathrm{C}$, resulting in a final substrate concentration of $3 \mu \mathrm{M}$ in $1 \times 10^{6}$ cells $/ \mathrm{ml}$. The reported Michaelis constant for the transporter $\left(K_{\mathrm{m}}\right)$ value of 11 drugs ranged from 0.18 to $76.7 \mu \mathrm{M}$ (Supplemental Table S2). The final free concentration of substrate in the presence of HSA would still be lower than $3 \mu \mathrm{M}$.

After incubation for 0.25 and 1.25 minutes, the reactions were terminated by separating the cells from the substrate solution. The hepatic uptake study was conducted using an oil-spin method described previously in detail (Miyauchi et al., 2018). The concentrations of medium and cells were analyzed by LC-MS/MS (Supplemental Table S1). Our results for the uptake clearances in the absence of HSA were comparable with reported values (Supplemental Table S2), indicating that the competition among drugs in the cassette mixture was minimal in our hepatic uptake experimental conditions.

Kinetic Analysis. The unbound fraction $\left(f_{\mathrm{u}}\right)$ for each drug in steady-state conditions using equilibrium dialysis was calculated using the following equation:

$$
\frac{1}{f_{\mathrm{u}}}=1+\frac{n[A l b]}{K_{\mathrm{d}}}
$$

where $n,[A l b]$ and $K_{\mathrm{d}}$ represent the number of binding sites on the albumin, the HSA concentration (micromolar), and the dissociation constant (micromolar) between drug and HSA, respectively. The $n$ values for some OATP1B1 substrates such as statins, antidiabetic drugs, and anti-HIV drugs have been reported to be 0.87-1.1 (Seedher and Kanojia, 2008; Gulati et al., 2009; Shi et al., 2017). For the simplicity of eq. 1 , the $n$ value was assumed to be unity. The molecular concentration of HSA was calculated using 66,500 as the molecular weight.

The initial uptake rates were obtained from the slopes of the time courses within $0.25-1.25$ minutes using linear regression analysis. The uptake clearances for total $\left(P S_{\text {inf }}\right)$ and unbound forms $\left(P S_{\mathrm{u}, \text { inf }}\right)$ of each drug were calculated by dividing the initial uptake velocity by the total and unbound drug concentrations in the incubation buffer at the various concentrations of HSA.

In our previous study, the "albumin-mediated" hepatic transport phenomenon was analyzed using the "facilitated-dissociation" model, in which the interaction between the cell surface and the drug-albumin complex enhances the dissociation of drug from the complex (Tsao et al., 1988a). A facilitation mechanism has been postulated to the effect that the interaction of the drug-albumin complex with the surface of hepatocytes induces a conformational change in albumin, resulting in enhanced dissociation of drug from the complex, providing unbound drug near the surface. Therefore, the uptake of highly albumin-bound drugs by hepatocytes includes the pathway of unbound drugs and the additional pathway of drugs dissociated from drug-albumin complexes near the surface. We assumed that unbound albumin and drug-bound albumin compete for the same binding site(s) on the surface of hepatocytes with the same affinity, which is the dissociation constant $\left(K_{\mathrm{d}, \mathrm{m}}\right)$ between albumin and the surface of hepatocytes. The fraction of albumin bound to the surface of the hepatocytes $(\lambda)$ is expressed as:

$$
\lambda=\frac{B_{\max }}{K_{\mathrm{d}, \mathrm{m}}+[A l b]}
$$

where $B_{\max }$ represents the capacity of albumin-binding sites on the surface of hepatocytes. The equations for the uptake rate $(v)$ of drug derived by Tsao et al. (1988a) are as follows:

$$
\begin{gathered}
v=P S_{\mathrm{inf}} \cdot c_{0}=P S_{\mathrm{u}, \text { inf }} \cdot f_{\mathrm{u}} \cdot c_{0}+P S_{\mathrm{b}, \mathrm{inf}} \cdot \lambda \cdot\left(1-f_{\mathrm{u}}\right) \cdot c_{0} \\
P S_{\mathrm{inf}}=P S_{\mathrm{u}, \text { inf }} \cdot f_{\mathrm{u}}+P S_{\mathrm{b}, \text { inf }} \cdot \lambda \cdot\left(1-f_{\mathrm{u}}\right)
\end{gathered}
$$

Substituting eqs. 1 and 2 into eq. 4 yields

$$
P S_{\mathrm{inf}}=P S_{\mathrm{u}, \text { inf }} \cdot \frac{K_{\mathrm{d}}}{K_{\mathrm{d}}+[A l b]}+P S_{\mathrm{b}, \text { inf }} \cdot \frac{B_{\max }}{K_{\mathrm{d}, \mathrm{m}}+[A l b]} \cdot \frac{[A l b]}{K_{\mathrm{d}}+[A l b]}
$$


where $c_{0}$ represents the total concentration of drug; $P S_{\mathrm{inf}}, P S_{\mathrm{u}, \mathrm{inf}}$, and $P S_{\mathrm{b}, \text { inf }}$ represent the uptake clearance for the total drug, unbound drug, and unbound drug dissociated from the drug-albumin complex at the surface, respectively. On the basis of the "facilitated-dissociation" model, the uptake clearance of unbound drug $\left(P S_{\text {inf (free) }}\right)$ and the uptake clearance of unbound drug associated from the drug-albumin complex $\left(P S_{\text {inf (bound) }}\right)$ at the cell surface were estimated from the first and second terms of the right-hand side of eq. 5, respectively, as follows.

$$
\begin{gathered}
P S_{\text {inf (free) }}=\frac{P S_{\mathrm{u}, \text { inf }} \cdot K_{\mathrm{d}}}{K_{\mathrm{d}}+[A l b]} \\
P S_{\text {inf (bound) }}=\frac{P S_{\mathrm{b}, \text { inf }} \cdot B_{\max }}{K_{\mathrm{d}, \mathrm{m}}+[A l b]} \cdot \frac{[A l b]}{K_{\mathrm{d}}+[A l b]}
\end{gathered}
$$

In eq. $7, P S_{\mathrm{b}, \text { inf }} \cdot B_{\max }$ represents the parameter of the clearance capacity for the uptake of "facilitated-dissociation" drugs, and is designated as $V_{\mathrm{B}, \max }$. The unbound uptake clearance in the presence of HSA, $\left(P S_{\mathrm{u}, \text { inf }}(+)\right)$, was calculated using the following equation modified from eqs. 1 and 5 .

$$
P S_{\mathrm{u}, \text { inf }}(+)=\frac{P S_{\mathrm{inf}}}{f_{u}}=P S_{\mathrm{u}, \mathrm{inf}}+\frac{V_{\mathrm{B} . \max } \cdot[A l b]}{K_{\mathrm{d}, \mathrm{m}}+[A l b]} \cdot \frac{1}{K_{\mathrm{d}}}
$$

This equation implies that $P S_{\mathrm{u}, \text { inf }}(+)$ shows saturation as $[A l b]$ increases. The $K_{\mathrm{d}}$ values of each compound were estimated using eq. 1 , and $P S_{\mathrm{u}, \text { inf }}, K_{\mathrm{d}, \mathrm{m}}$, and $V_{\mathrm{B}, \max }$ were obtained with eq. 5 using nonlinear least-squares fitting software (Napp, version 2.31, The University of Tokyo Hospital, Japan) (Hisaka and Sugiyama, 1998).

Extrapolation to In Vivo Hepatic Clearances from the In Vitro Uptake Clearance by Suspended Hepatocytes. The in vitro unbound hepatic uptake clearance (microliters per $10^{6}$ cells per milliliter) was scaled up to in vivo (milliliters per minute per kilogram body weight) using the following physiologic scaling factors: $1.2 \times 10^{8}$ cells/g liver and $25.7 \mathrm{~g}$ liver $/ \mathrm{kg}$ body weight (Davies and Morris, 1993; Miyauchi et al., 1993).

In vivo hepatic overall intrinsic clearance, representing the elimination of unbound drug $\left(C L_{\mathrm{h}, \mathrm{u}, \mathrm{int}, \mathrm{all}}^{\mathrm{vivo}}\right)$ from circulating blood, was calculated from the reported in vivo hepatic clearance (Supplemental Table S3) using the dispersion model (eqs. 9-12) (Roberts and Rowland, 1986) and the well-stirred model (eqs. 9 and 13) (Pang and Rowland, 1977).

$$
C L_{\mathrm{h}, \mathrm{B}}=Q_{\mathrm{h}} \times\left(1-F_{\mathrm{h}}\right)
$$

For the dispersion model:

$$
\begin{gathered}
F_{\mathrm{h}}=\frac{4 a}{(1+a)^{2} \cdot \exp \left\{\frac{(a-1)}{2 D_{\mathrm{N}}}\right\}-(1-a)^{2} \cdot \exp \left\{-\frac{(a+1)}{2 D_{\mathrm{N}}}\right\}} \\
a=\left(1+4 R_{\mathrm{N}} \cdot D_{\mathrm{N}}\right)^{1 / 2} \\
R_{\mathrm{N}}=f_{\mathrm{B}} \cdot C L_{\mathrm{h}, \mathrm{u}, \text { int,all }}^{\mathrm{vivo}}
\end{gathered}
$$

For the well-stirred model:

$$
F_{\mathrm{h}}=Q_{\mathrm{h}} /\left(Q_{\mathrm{h}}+f_{\mathrm{B}} \cdot C L_{\mathrm{h}, \mathrm{u}, \text { int,all }}^{\text {vivo }}\right)
$$

Where $C L_{\mathrm{h}, \mathrm{B}}, Q_{\mathrm{h}}, D_{\mathrm{N}}$, and $f_{\mathrm{B}}$ are the hepatic clearance, hepatic blood flow rate (20.7 $\mathrm{ml} / \mathrm{min}$ per kilogram body weight) (Davies and Morris, 1993), the dispersion number (0.17) (Roberts and Rowland, 1986), and the unbound fraction in the blood, respectively. For almost all of the drugs used, it has been suggested that hepatic uptake is a rate-determining step for hepatic elimination (Watanabe et al., 2010; Izumi et al., 2017). According to the extended clearance concept, in vivo hepatic intrinsic clearance for 11 OATP substrates could be well accounted for by the in vitro hepatic uptake clearance, regardless of the involvement of hepatic metabolism.

\section{Results}

Unbound Fractions in Human Plasma and HSA. We determined the unbound fraction $\left(f_{\mathrm{p}}\right)$ of 11 drugs in human plasma. These values were used for the calculation of blood unbound fraction as $f_{\mathrm{p}} / R_{\mathrm{B}}$ (where $R_{\mathrm{B}}$ is the blood partitioning; Table 2). The $f_{\mathrm{u}}$ values were determined in the presence of HSA at six concentrations $(0 \%, 0.125 \%$, $0.25 \%, 0.5 \%, 1 \%$, and $5 \%$ ). The $f_{\mathrm{p}}$ values for almost all drugs except RSV and PRV were less than 0.05 (0.000787-0.0308), whereas those for RSV and PRV were 0.134 and 0.563 , respectively. The $f_{\mathrm{p}}$ values were very close to $f_{\mathrm{u}}$ values in the presence of $5 \%$ HSA, a normal albumin concentration (Table 2). The $K_{\mathrm{d}}$ values of drugs were obtained by nonlinear least-squares fitting using eq. 1 (Fig. 1). For nine of the drugs, these were $0.490-27.1 \mu \mathrm{M}$, and for RSV and PRV they were 94.5 and $667 \mu \mathrm{M}$, respectively (Table 1). Most of the drugs used here are highly bound to albumin in plasma (bound form $>95 \%$ ), whereas two drugs, RSV and PRV, showed low protein binding, with $f_{\mathrm{p}}$ values of 0.134 for RSV and 0.563 for PRV (Fig. 1; Table 1). These results were consistent with the $f_{\mathrm{p}}$ values reported previously (Colussi et al., 1997; Hatorp, 2002; Blanchard et al., 2005; Watanabe et al., 2010, 2011), indicating that the major binding protein in the plasma is indeed albumin. The recovery of the measurement for 11 drugs in the uptake experiments was more than $92 \%$, irrespective of HSA in the buffer. The nonspecific adsorption to the wall of test tubes was assumed to be minimal.

Effect of Albumin on Uptake Clearances by Isolated Human Hepatocytes. The $P S_{\text {inf }}$ (hepatic uptake clearance for total drug) values of drugs at various concentrations of HSA $(0.125 \%, 0.25 \%$, $0.5 \%$, and $1 \%$ ) were determined from the initial slopes of the uptake volume (Supplemental Fig. S1). However, the $P S_{\text {inf }}$ value in the presence of $5 \%$ HSA could not be determined robustly because of the avid binding of these drugs to albumin, resulting in no significant time-dependent uptake volume by hepatocytes over $0.25-1.25$ minutes. The $P S_{\mathrm{u} \text {,inf }}(+)$ (the hepatic unbound uptake clearance in the presence of the albumin) value was estimated by dividing the $P S_{\text {inf }}$ value by the $f_{\mathrm{u}}$ value at the various concentrations of HSA, whereas the $P S_{\mathrm{u} \text {,inf }}$ value was obtained in the absence of HSA. The $P S_{\text {inf }}$ values of almost all drugs except PRV were decreased in association with increases in the concentration of HSA, whereas the $P S_{\mathrm{u} \text {,inf }}(+)$ values were increased substantially (Figs. 2 and 3; Supplemental Fig. S2), thus demonstrating that these 10 drugs with high binding to the albumin show "albuminmediated" hepatic uptake. Nevertheless, PRV with its low protein binding to albumin $\left(f_{\mathrm{p}}=0.563\right)$ did not show any change in the $P S_{\mathrm{inf}}$ and $P S_{\mathrm{u}, \text { inf }}(+)$ values, irrespective of the concentration of HSA (Fig. 3; Supplemental Fig. S2). Furthermore, the relationships between the $P S_{\text {inf }}$ values and HSA concentrations for the 10 drugs except for PRV were simultaneously fitted to eq. 5 on the basis of the "facilitated-dissociation" model (Tsao et al., 1988a), and the estimated $K_{\mathrm{d}, \mathrm{m}}$ value was $45.2 \pm$ $13.0 \mu \mathrm{M}$ ( \pm calculated S.D.). The $P S_{\mathrm{u} \text {,inf }}$ values obtained in the absence of HSA were very close to those of $P S_{\mathrm{u}, \mathrm{inf}}$ estimated in the fitting analysis by the "facilitated-dissociation" model. These estimated parameters are summarized in Table 1 . The $P S_{\text {inf values in the presence }}$ of a normal physiologic albumin concentration (5\%) were predicted according to eq. 5 by the "facilitated-dissociation" model. The predicted $P S_{\text {inf }}$ values were much greater than those obtained on the basis of the "free drug" hypothesis (Fig. 3). The contributions of "albuminmediated" uptake clearance $\left(P S_{\text {inf (bound) }}\right)$ to total uptake clearance for these 10 drugs were estimated to be 55-98\%, according to eqs. 6 and 7 (Table 1).

Extrapolation to In Vivo Hepatic Clearances from In Vitro Uptake Data. The $P S_{\mathrm{u} \text {,inf }}$ values of 10 drugs were extrapolated to the $P S_{\mathrm{u}, \text { inf }}^{\mathrm{vitro}}$ values $\left(\mathrm{ml} / \mathrm{min} / \mathrm{kg}\right.$ body weight). As shown in Table 2 , the $f_{\mathrm{B}}$. $P S_{\mathrm{u}, \text { inf }}^{\mathrm{vitr}}$ values whose basis was the "free drug" hypothesis were underestimated substantially compared with the $f_{\mathrm{B}} \cdot C L_{\mathrm{h}, \mathrm{u} \text {,int,all }}^{\text {vivo }}$ (the in vivo unbound overall hepatic intrinsic clearance) values obtained from hepatic clearance by the dispersion model (Fig. 4A) and the well-stirred model (Fig. 5A). The $P S_{\mathrm{u}, \text { inf }}(+)$ values for 10 drugs at 

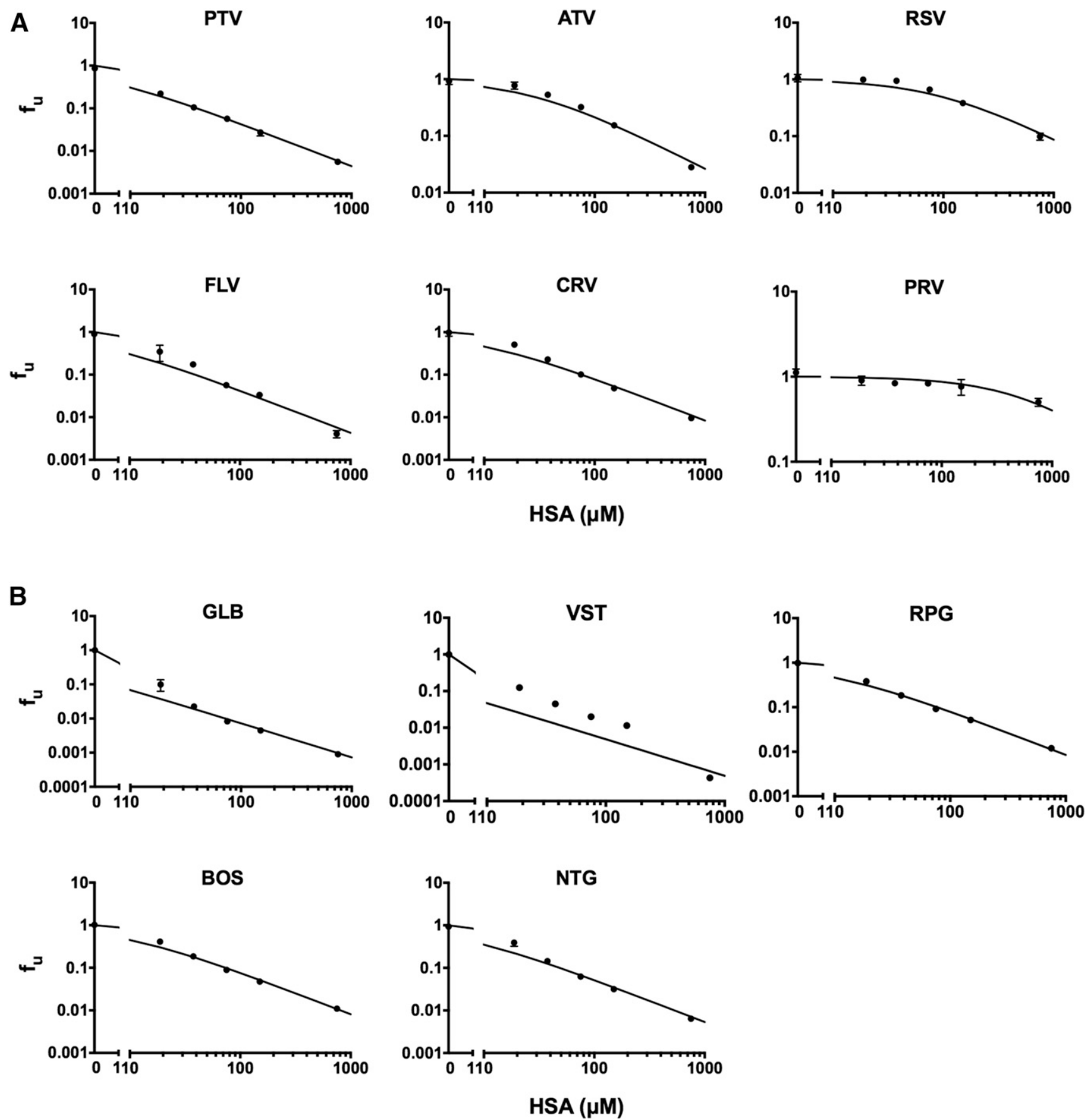

Fig. 1. The unbound fraction $\left(f_{\mathrm{u}}\right)$ of 11 drugs in HSA at $0 \%, 0.125 \%, 0.25 \%, 0.5 \%, 1 \%$, and $5 \%$. The filled circles represent the observed HSA $f_{\mathrm{u}}$ values $(\mathrm{mean} \pm \mathrm{S}$.D., $n=4)$ in the absence and presence of HSA, which concentrations were 18.8, 37.6, 75.2, 150, and $752 \mu \mathrm{M}$. The solid lines represent the fitted line estimated from eq. 1. (A) and (B) show sets A and B, respectively. Key: PTV, pitavastatin; ATV, atorvastatin; FLV, fluvastatin; CRV, cerivastatin; GLB, glibenclamide; VST, valsartan; RPG, repaglinide; BOS, bosentan; NTG, nateglinide.

$5 \%$ HSA estimated on the basis of the "facilitated-dissociation" model (eq. 8) were also extrapolated to the in vivo value per body weight $\left(P S_{\mathrm{u}, \text { inf }}^{\text {vitro }}, \mathrm{ml} / \mathrm{min} / \mathrm{kg}\right)($ Table 2$)$. The $f_{\mathrm{B}} \cdot P S_{\mathrm{u}, \text { inf }}^{\mathrm{vitro}}$ values at $5 \%$ HSA

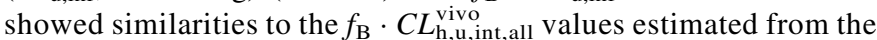
hepatic clearance regardless of the dispersion or well-stirred model (Figs. 4B and 5B; Table 2). The "albumin-mediated" uptake factors or enhancement of the hepatic uptake clearance $(R)$ in the physiologic albumin concentration range were estimated as the ratios of $P S_{\mathrm{u}, \text { inf }}(+)$ values at $5 \%$ HSA to $P S_{u \text {,inf }}$ values in the absence of HSA; the estimated $R$ values were $2.44-63.8$ (Table 2).
As shown in Figs. 4B and 5B, the "albumin-mediated" uptake factor improved the prediction of IVIVE, but the relationship between $f_{\mathrm{B}} \cdot P S_{\mathrm{u}, \text { inf }}^{\mathrm{vitro}}$ and $f_{\mathrm{B}} \cdot C L_{\mathrm{h}, \mathrm{u} \text {,int,all }}^{\mathrm{vivo}}$ still showed some underestimation in the IVIVE from the in vitro hepatic uptake clearance. The ratios of $f_{\mathrm{B}} \cdot C L_{\mathrm{h}, \mathrm{u}, \text { int,all }}^{\mathrm{vivo}}$ whose basis was the dispersion and wellstirred models to $f_{\mathrm{B}} \cdot P S_{\mathrm{u}, \text { inf }}^{\mathrm{vitr}}$ at $5 \%$ HSA were $0.63-5.57$ and $0.63-7.11$, respectively, and the average value was 2.44 and 3.19, respectively (Table 2). Furthermore, the $f_{\mathrm{B}} \cdot P S_{\mathrm{u} \text {,inf values at } 5 \% \text { HSA }}^{\mathrm{vitro}}$ were corrected by the mean value (2.44 and 3.19) designated as scaling factor $(S F)$, giving a better relationship between $f_{\mathrm{B}} \cdot P S_{\mathrm{u}, \text { inf }}^{\mathrm{vitro}}$. 
TABLE 1

Unbound fractions in plasma and 5\% HSA, and the estimated contribution of "albumin-mediated" uptake of 11 drugs based on Tsao's model

Data are presented as the mean or mean \pm S.D.

\begin{tabular}{|c|c|c|c|c|c|c|c|c|c|c|}
\hline \multirow{2}{*}{ Drugs } & \multirow{2}{*}{$f_{\mathrm{p}}$} & \multirow{2}{*}{$f_{\mathrm{u}, 5 \% \text { HSA }}$} & \multirow{2}{*}{$\mathrm{K}_{\mathrm{d}}^{\mathrm{a}}$} & \multicolumn{2}{|c|}{$P S_{\mathrm{u}, \mathrm{inf}}$} & \multirow{2}{*}{$K_{\mathrm{d}, \mathrm{m}}{ }^{\mathrm{d}}$} & \multirow{2}{*}{$V_{\mathrm{B}, \max }{ }^{\mathrm{c}}$} & \multicolumn{2}{|c|}{$P S_{\mathrm{inf}}$} & \multirow{2}{*}{$\begin{array}{c}\text { Contribution (\%) } \\
\text { of "albumin-mediated" uptake }\end{array}$} \\
\hline & & & & Observed $^{\mathrm{b}}$ & Calculated $^{\mathrm{c}}$ & & & Free $^{\mathrm{e}}$ & Bound $^{\mathrm{d}}$ & \\
\hline & & & $\mu M$ & \multicolumn{2}{|c|}{$\mu \mathrm{l} / \mathrm{min} / 10^{6}$ cells } & $\mu M$ & $\mu M$ & \multicolumn{2}{|c|}{$\mu \mathrm{l} / \mathrm{min} / 10^{6}$ cells } & \\
\hline Pitavastatin & 0.00541 & 0.00558 & $4.44 \pm 0.21$ & $36.2 \pm 11.4$ & $36.3 \pm 3.1$ & $45.2 \pm 13.0$ & $225 \pm 79$ & 0.213 & 0.281 & 56.9 \\
\hline Atorvastatin & 0.0308 & 0.0281 & $27.1 \pm 3.2$ & $24.2 \pm 3.7$ & $23.5 \pm 2.5$ & & $1090 \pm 258$ & 0.842 & 1.32 & 61.0 \\
\hline Rosuvastatin $^{\mathrm{g}}$ & 0.134 & 0.0988 & $94.5 \pm 12.1$ & $4.01 \pm 1.61$ & $3.76 \pm 0.58$ & & $480 \pm 145$ & 0.448 & 0.535 & 54.4 \\
\hline Fluvastatin & 0.00428 & 0.00405 & $4.33 \pm 0.75$ & $62.1 \pm 22.9$ & $62.4 \pm 4.1$ & & $1090 \pm 193$ & 0.356 & 1.36 & 79.3 \\
\hline Cerivastatin & 0.00734 & 0.00961 & $8.48 \pm 0.93$ & $77.5 \pm 23.3$ & $74.1 \pm 4.56$ & & $1160 \pm 239$ & 0.864 & 1.45 & 62.6 \\
\hline Pravastatin & 0.563 & 0.502 & $667 \pm 121$ & $3.55 \pm 2.17$ & NA & & NA & NA & NA & NA \\
\hline Glibenclamide & 0.000787 & 0.000897 & $0.730 \pm 0.104$ & $32.2 \pm 4.7$ & $32.3 \pm 3.0$ & & $98.9 \pm 35.7$ & 0.0312 & 0.124 & 79.9 \\
\hline Valsartan & 0.00165 & 0.000431 & $0.490 \pm 0.210$ & $1.87 \pm 0.65$ & $1.87 \pm 0.72$ & & $40.1 \pm 18.3$ & 0.00122 & 0.0502 & 97.6 \\
\hline Repaglinide & 0.00676 & 0.0120 & $8.55 \pm 0.44$ & $39.2 \pm 10.7$ & $40.0 \pm 3.3$ & & $1640 \pm 271$ & 0.441 & 2.04 & 82.2 \\
\hline Bosentan & 0.00814 & 0.0110 & $8.16 \pm 0.55$ & $23.2 \pm 5.7$ & $23.4 \pm 2.5$ & & $796 \pm 157$ & 0.249 & 0.988 & 79.8 \\
\hline Nateglinide & 0.00497 & 0.00641 & $5.39 \pm 0.58$ & $12.5 \pm 3.0$ & $12.7 \pm 1.8$ & & $155 \pm 57$ & 0.0886 & 0.193 & 68.5 \\
\hline
\end{tabular}

$f_{\mathrm{p}}$, plasma-unbound fraction; $f_{\mathrm{u}}$, HSA-unbound fraction; HSA, human serum albumin; $K_{\mathrm{d}}$, the dissociation constant between drug and HSA; $K_{\mathrm{d}, \mathrm{m}}$, the dissociation constant for the

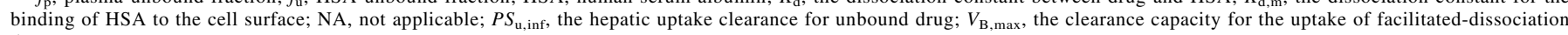
drugs.

${ }^{a}$ These values were obtained from in vitro HSA binding experiments for each drug by eq. 1, and they were fixed when fitting with Tsao's model

${ }^{b}$ These values were observed in the suspended hepatocyte uptake study at $0 \%$ HSA.

${ }^{c}$ These values were estimated using Tsao's model (eq. 5) and the observed $P S_{\text {inf }}$ at the various concentration of HSA

${ }^{d}$ Uptake clearance by bound form at $5 \%$ HSA, calculated using eq. 7.

${ }^{e}$ Uptake clearance by the "free drug" theory at $5 \%$ HSA, calculated using eq. 6.

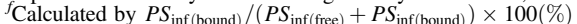

${ }^{g} P S_{\mathrm{u}, \text { inf }}$ and $V_{\mathrm{B}, \max }$ for rosuvastatin were estimated using Tsao's model with a fixed value $(45.2 \mu \mathrm{M})$ of $K_{\mathrm{d}, \mathrm{m}}$

$S F$ and $f_{\mathrm{B}} \cdot C L_{\mathrm{h}, \mathrm{u}, \text { int,all }}^{\mathrm{vivo}}$ within a 3 -fold range of difference regardless of estimated models of $f_{\mathrm{B}} \cdot C L_{\mathrm{h}, \mathrm{u}, \text { int,all }}^{\mathrm{vivo}}$ (Fig. 4C; Table 2).

\section{Discussion}

Human-derived reagents, such as hepatic microsomes, cytosols and suspended hepatocytes, are now available for in vitro studies (Chiba et al., 2009; Shitara et al., 2013), resulting in success in the prediction of hepatic clearances for new chemical entities at early stages of drug discovery and development. Here, we investigated whether the in vitro hepatic uptake clearance by suspended human hepatocytes could be extrapolated quantitatively to estimate the in vivo hepatic clearances for 11 drugs known as OATP substrates.

Previously, in vitro hepatic uptake clearance with cryopreserved human hepatocytes showed good prediction of in vivo hepatic clearance for some OATP substrates (Watanabe et al., 2010, 2011; Kimoto et al., 2012). Nevertheless, the predictions of human hepatic clearances in some anionic drugs with high binding to albumin were poor, showing underestimation of IVIVE for hepatic uptake clearances. Consistent with this notion, Figs. 4A and 5A show the underestimation of IVIVE for the hepatic uptake clearances regardless of estimated models of $f_{\mathrm{B}} \cdot C L_{\mathrm{h}, \mathrm{u} \text {,int,all }}^{\mathrm{vivo}}$. We hypothesized that "albumin-mediated" hepatic uptake would result in poor predictions of IVIVE because uptake studies in human hepatocytes have usually been conducted in the absence of serum albumin. Recently, using primary cultured rat hepatocytes and suspended human hepatocytes, we demonstrated that IVIVE for hepatic clearance for Oatp/OATP substrates with high protein binding was robustly improved by an "albumin-mediated" hepatic uptake mechanism considered in the analysis (Miyauchi et al., 2018), thereby suggesting that the underestimation in IVIVE could be attributed to an "albumin-mediated" hepatic uptake mechanism. In the present study, we further investigated the effect of HSA on the hepatic uptake clearance of 11 anionic OATP substrates by using suspended human hepatocytes in the absence and presence of various concentrations of HSA, and the improvement in the prediction of quantitative IVIVE for hepatic uptake clearance values by considering "albumin-mediated" hepatic uptake mechanisms that used the "facilitated-dissociation" model as a basis.

The $P S_{\text {inf }}$ values of 11 drugs by human hepatocytes were determined in the presence of HSA, and the $P S_{\mathrm{u} \text {,inf }}(+)$ values were obtained by dividing the $P S_{\text {inf }}$ by $f_{\mathrm{u}}$ for the various concentrations of HSA. Except for PRV, all drugs showed decreases in the $P S_{\text {inf }}$ values along with increases in HSA concentration (Figs. 2 and 3), whereas there was a substantial increase in $P S_{\mathrm{u} \text {,inf }}(+)$ value (Fig. 2B; Supplemental Fig. S2), which invalidated the "free drug" hypothesis, suggesting that "albumin-
A

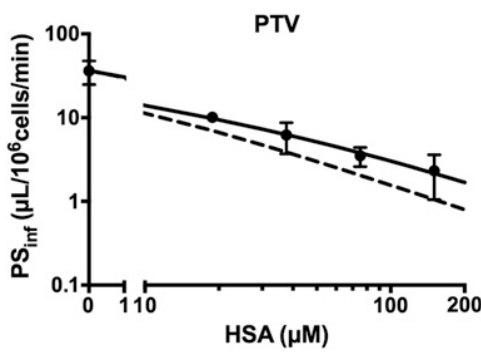

B

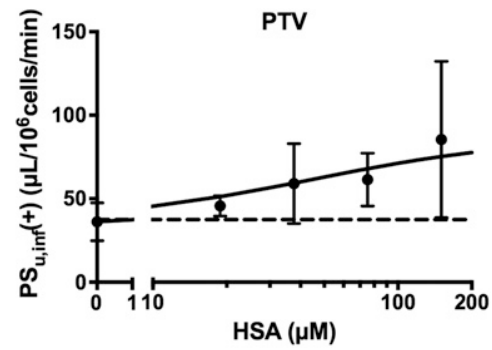

Fig. 2. The uptake clearances for total form $\left(P S_{\text {inf }}\right)(A)$ and the unbound uptake clearance in the presence of $\operatorname{HSA}\left[P S_{u \text {,inf }}(+)\right](\mathrm{B})$ of pitavastatin (PTV) in suspended human hepatocytes. The filled circles, solid lines, and broken lines represent the observed or calculated values (mean \pm S.D., $n=8$ ), the fitted line by Tsao's model, and the theoretical line with the "free drug" theory as a basis, respectively. 


\section{A}
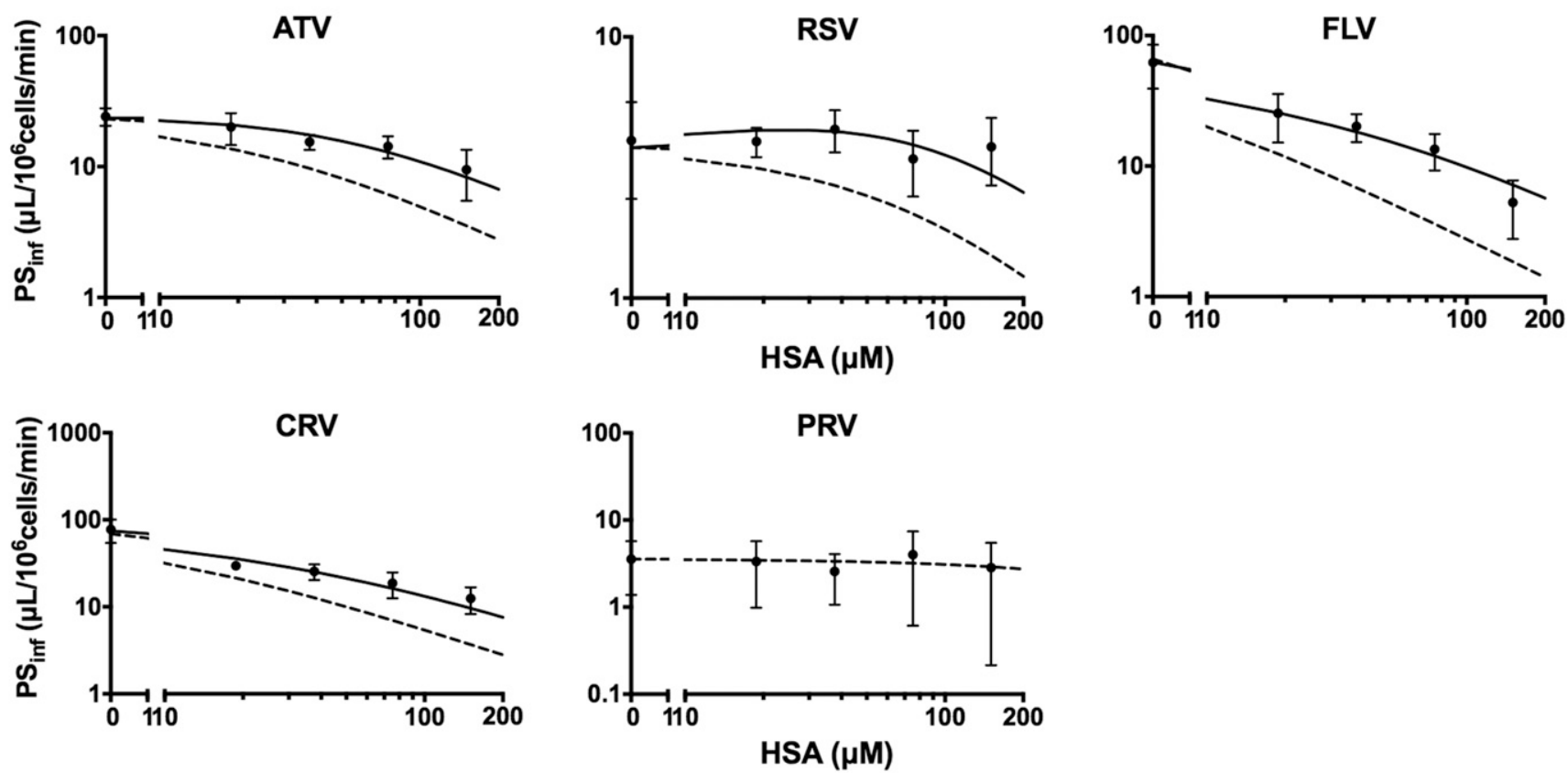

B
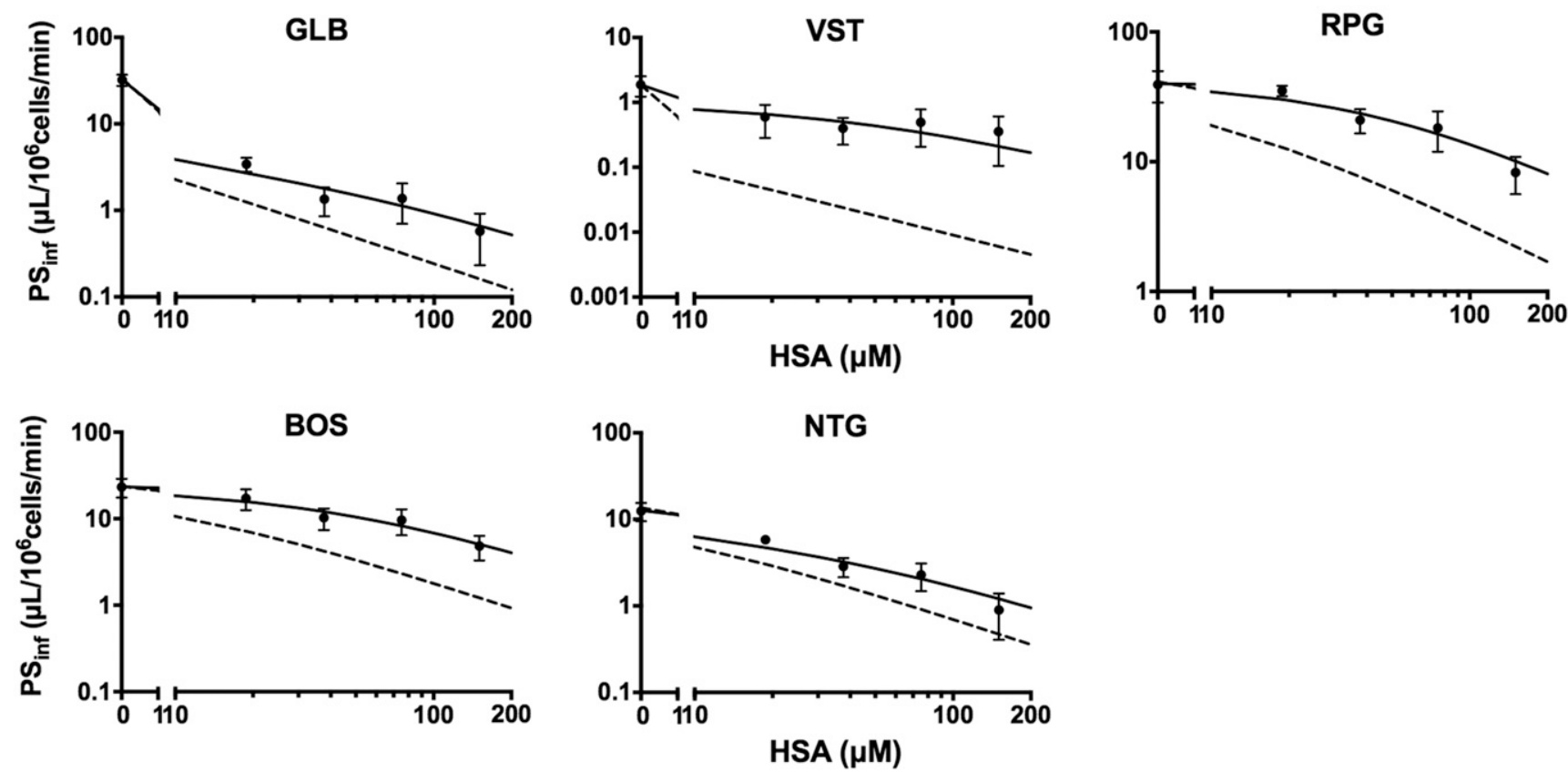

Fig. 3. The observed uptake clearances for total form $\left(P S_{\text {inf }}\right)$ of clinical OATP substrates in suspended human hepatocytes. The filled circles, solid lines, and broken lines represent the observed $P S_{\text {inf }}$ (mean \pm S.D., $n=8$ ), the fitted line by Tsao's model (eq. 5), and the theoretical line with the "free drug" theory as a basis, respectively. (A) and (B) represent sets A and B, respectively. Key as in Fig. 1. Notes: 1) When fitting for RSV by Tsao's model, only $P S_{\mathrm{u}, \text { inf }}$ and $V_{\mathrm{B}, \max }$ were estimated with a fixed $K_{\mathrm{d}, \mathrm{m}}$ value (Table 1 ). 2) The fitting by Tsao's model was not performed for PRV.

mediated" hepatic uptake for various OATP substrates with high protein binding was also observed in human hepatocytes. Nevertheless, PRV with low protein binding to albumin $\left(f_{\mathrm{p}}=0.563\right)$ did not show any change in the $P S_{\mathrm{inf}}$ and $P S_{\mathrm{u} \text {,inf }}(+)$ values, irrespective of the concentrations of HSA (Fig. 3; Supplemental Fig. S2).

In the present study, the $P S_{\mathrm{u} \text {,inf }}(+)$ was composed of the uptake clearances for unbound drug and unbound drug dissociated from the drugalbumin complex near the cell surface (eq. 8). If "albumin-mediated" uptake is involved in hepatic uptake, the $P S_{\mathrm{u}, \mathrm{inf}}(+)$ value would be greater than the $P S_{\mathrm{u} \text {,inf }}$ value by the uptake clearance of unbound drug dissociated from the drug-albumin complex. It should be noted that $P S_{\mathrm{u}, \text { inf }}(+)$ is a hybrid parameter accounting for the "albumin-mediated" uptake clearance attributable to the hepatic uptake of unbound drug dissociated from the drug-albumin complex.

Previously, the hepatic uptake of a number of endogenous and exogenous ligands and OATP substrates has been shown to exhibit the 
Kim et al.

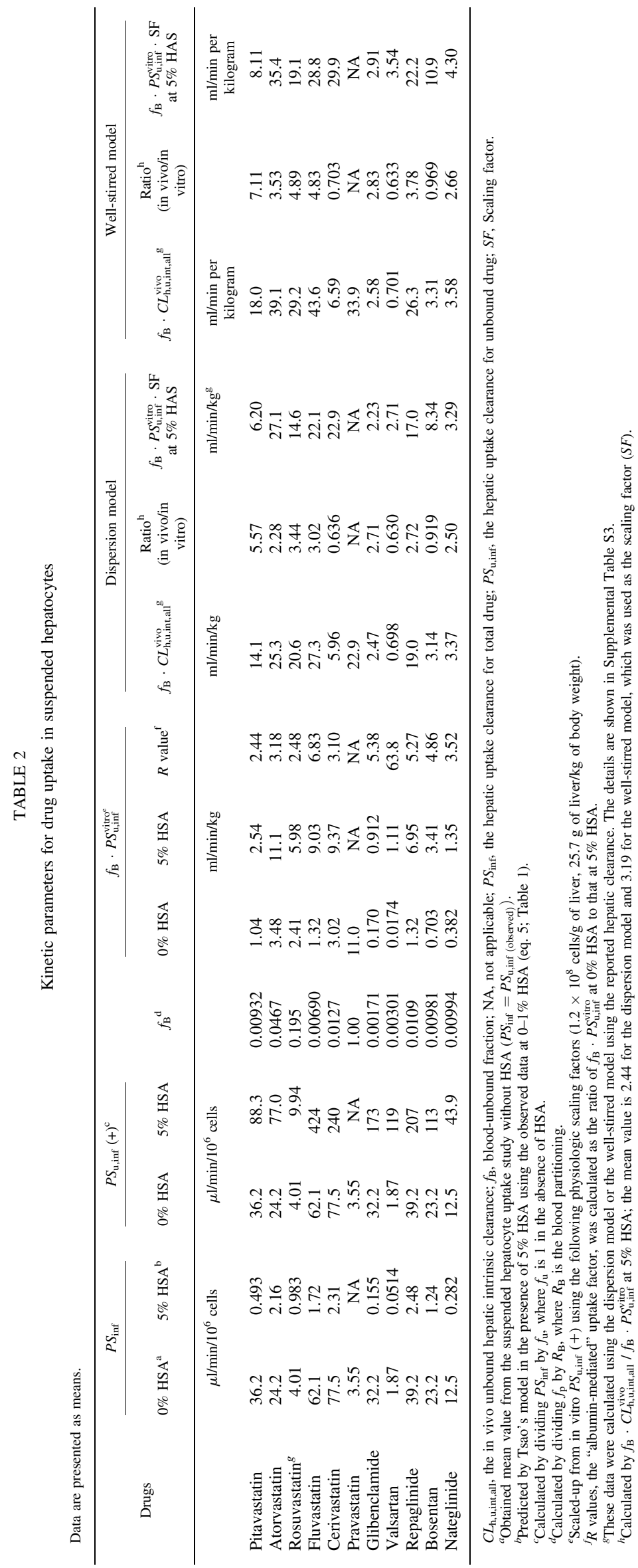



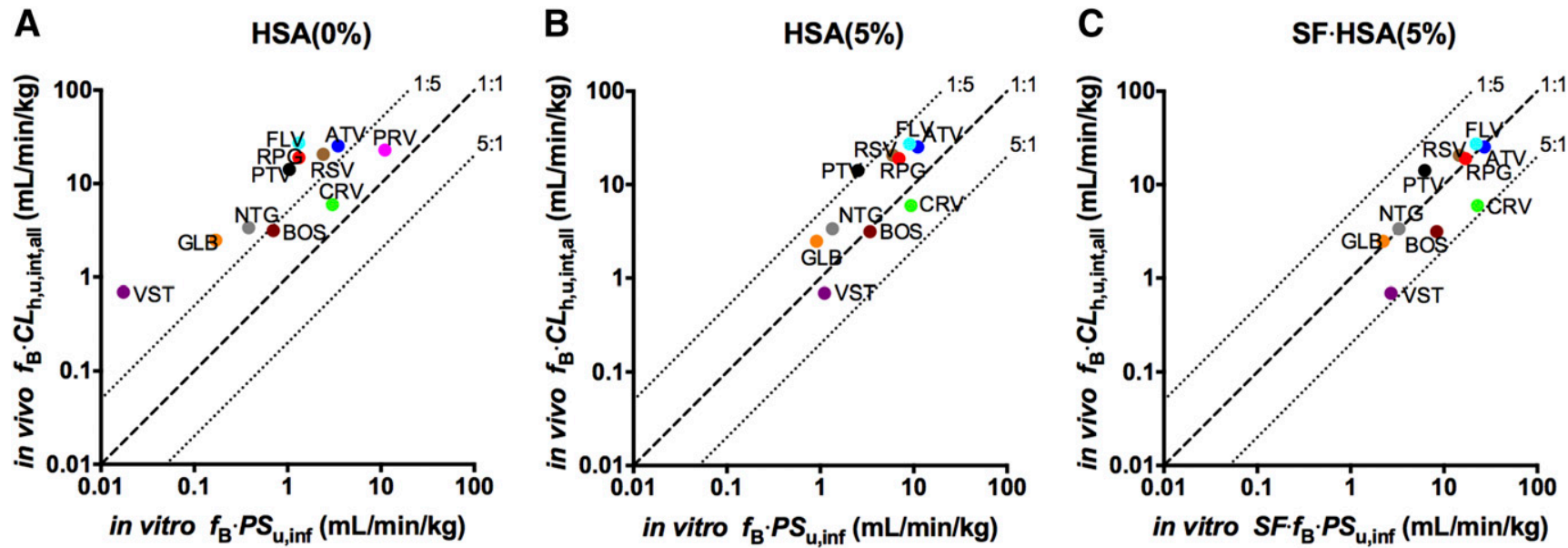

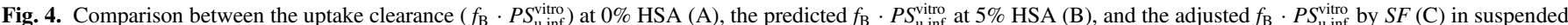
human hepatocytes and the observed hepatic overall intrinsic clearance $\left(f_{\mathrm{B}} \cdot C L_{\mathrm{h}, \mathrm{u} \text { int all }}^{\text {vilo }}\right)$ by the dispersion model. The $f_{\mathrm{B}} \cdot P S_{\mathrm{u}, \text { inf }}^{\text {vitro }}$ at $5 \%$ HSA was predicted by Tsao's model, the adjusted $f_{\mathrm{B}} \cdot P S_{\mathrm{u}, \text { inf }}^{\text {vitro }}$ at $5 \% \mathrm{HSA}$ was calculated by the predicted $f_{\mathrm{B}} \cdot P S_{\mathrm{u}, \text { inf }}^{\text {vitro }}$, and $S F$ is 2.44 , the average value of the ratios of $f_{\mathrm{B}} \cdot C L_{\mathrm{h}, \mathrm{u}, \mathrm{int}, \text { all }}^{\text {vivo }}$ to $f_{\mathrm{B}} \cdot P S_{\mathrm{u}, \text { inf }}^{\text {vitro }}$ at $5 \%$ HSA. The dashed and dotted lines represent the line of unity and the lines of the 1:5 and 5:1 correlations, respectively. Key as in Fig. 1.

kinetics of "albumin-mediated" hepatic transport (Weisiger et al., 1981, 1984; Forker et al., 1982; Forker and Luxon, 1983; Tsao et al., 1986, 1988b; Pond et al., 1992; Fujino et al., 2018; Miyauchi et al., 2018). We have analyzed the "albumin-mediated" hepatic uptake transport, which uses the "facilitated-dissociation" model as a basis, in which the interaction of the drug-albumin complex with the surface of hepatocytes enhances the dissociation of the drug from the complex and provides unbound drug that is more available for hepatic uptake (Tsao et al., 1988a). The relationships between the $P S_{\text {inf }}$ values and HSA concentrations in all 10 drugs except PRV were simultaneously fitted to eq. 5 . $K_{\mathrm{d}, \mathrm{m}}$, a drug-independent parameter showing the dissociation constant between the albumin (unbound albumin or drug-bound albumin) and the surface of hepatocytes, was estimated to be $45 \mu \mathrm{M}$ regardless of the drug tested, which is consistent with results reported in other studies (Weisiger et al., 1981; Forker and Luxon, 1983). $V_{\mathrm{B}, \max }$, a drugdependent parameter showing the clearance capacity for the uptake of "facilitated-dissociation" drug, was estimated at various values (Table 1). The $P S_{\text {inf }}$ values at the normal blood concentration of HSA $(5 \%)$ are a key parameter of the quantitative IVIVE of hepatic uptake clearances; unfortunately, these values could not be determined robustly because the drugs bind avidly to the albumin, resulting in no time-dependent uptake volume by hepatocytes during $0.25-1.25$ minutes in the presence of 5\% HSA. To predict IVIVE quantitatively for hepatic uptake clearances by isolated hepatocytes, we therefore estimated $P S_{\text {inf }}$ in the presence of $5 \%$ HSA according to eq. 5 on the basis of the "facilitateddissociation" model (Table 2). The $P S_{\text {inf }}$ values with the "facilitateddissociation" model as a basis were higher than values whose basis was the "free drug" hypothesis (Fig. 3; Table 2). The contributions of "albumin-mediated" uptake to the total uptake clearance of 10 drugs were $54 \%-98 \%$, whereas rosuvastatin with its relatively low protein binding property $\left(0.134\right.$ of $\left.f_{\mathrm{p}}\right)$ exhibited the minimum value for "albumin-mediated" hepatic uptake contribution (Table 1). In light of these findings, albumin facilitates the hepatic uptake of the bound form of OATP substrate drugs with high protein binding (approximately $>90 \%$ ), a major pathway of hepatic uptake in the normal range of albumin concentration in the blood, thereby violating the "free drug" hypothesis. Therefore, we believe that the "albumin-mediated" hepatic uptake mechanism may improve the prediction accuracy for IVIVE.
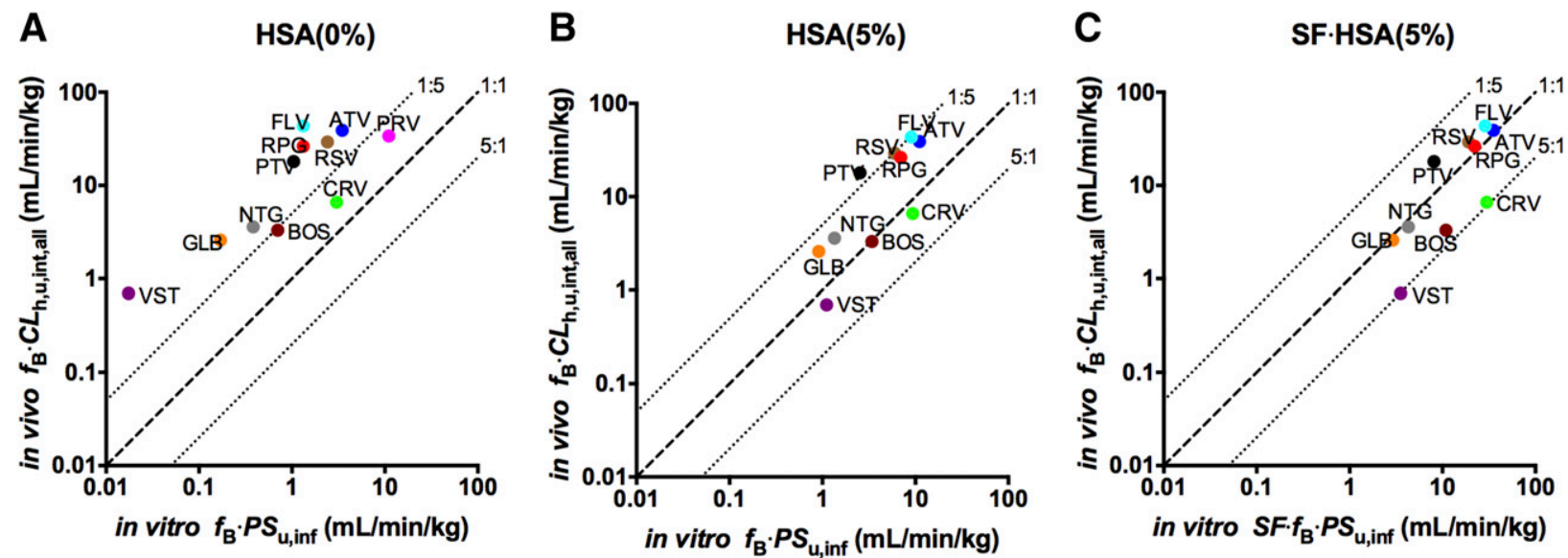

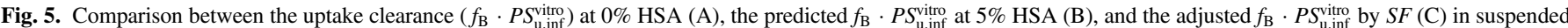

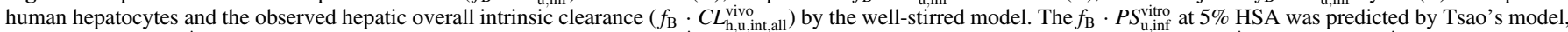

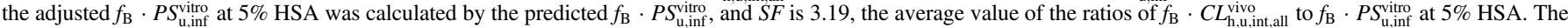
dashed and dotted lines represent the line of unity and the lines of the 1:5 and 5:1 correlations, respectively. Key as in Fig. 1. 
Regardless of estimated models of $f_{\mathrm{B}} \cdot C L_{\mathrm{h}, \mathrm{u} \text {,int,all }}^{\mathrm{vivo}}$, the $f_{\mathrm{B}} \cdot P S_{\mathrm{u}, \text { inf }}^{\mathrm{vitro}}$ values estimated from in vitro $P S_{\mathrm{u}, \text { inf }}(+)$ values at $5 \%$ HSA correlated better with the $f_{\mathrm{B}} \cdot C L_{\mathrm{h}, \mathrm{u} \text {,int,all }}^{\text {vivo }}$ values estimated from the hepatic clearances (Fig. 4B; Fig. 5B; Table 2), suggesting that the underestimation of IVIVE for the hepatic uptake clearances could be adjusted sufficiently by "albumin-mediated" uptake factors, the ratio of $f_{\mathrm{B}} \cdot P S_{\mathrm{u} \text {,inf }}^{\mathrm{vitro}}$ at $0 \% \mathrm{HSA}$ to that at $5 \% \mathrm{HSA}(R)$, or by enhancement of the hepatic uptake clearance in the normal range of albumin concentrations; the $R$ values were estimated to be 2.44-63.8 (Table 2). Nevertheless, the relationship between $f_{\mathrm{B}} \cdot P S_{\mathrm{u}, \text { inf }}^{\mathrm{vitr}}$ and $f_{\mathrm{B}} \cdot C L_{\mathrm{h}, \mathrm{u} \text {,int,all }}^{\text {vivo }}$ continued to show slight underestimation of IVIVE from the in vitro hepatic uptake clearances, although the "albumin-mediated" uptake factor improved the prediction. The ratios of $f_{\mathrm{B}} \cdot C L_{\mathrm{h}, \mathrm{u} \text {,int,all }}^{\mathrm{vivo}}$ with the dispersion and wellstirred models as bases to $f_{\mathrm{B}} \cdot P S_{\mathrm{u}, \text { inf }}^{\mathrm{vitro}}$ at 5\% HSA were 0.63-5.57 and $0.63-7.11$, respectively, and the mean values were 2.44 and 3.19, respectively (Table 2). The ratios of the in vivo-to-in vitro for hepatic clearance exhibited substrate dependency. Although drugs are only taken up by OATP1B1, the affinities for OATP1B1 of each drug ranged widely from low to high (the reciprocal of the $K_{\mathrm{m}}$ values, summarized in Supplemental Table S2). Furthermore, the relationship between the values of "albumin-mediated" uptake factors $(R)$ and the affinities of drug for OATP1B1 exhibited a tendency by which the higher the affinity for the transporter, the more albumin effectively enhanced uptake (Miyauchi et al., 2018). Furthermore, the $f_{\mathrm{B}} \cdot P S_{\mathrm{u} \text {,inf }}^{\mathrm{vitro}}$ values at $5 \%$ HSA were corrected by the mean value designated as $S F$, resulting in a better relationship between $f_{\mathrm{B}} \cdot P S_{\mathrm{u} \text {,inf }}^{\mathrm{vitro}} \cdot \mathrm{SF}$ and $f_{\mathrm{B}} \cdot C L_{\mathrm{h}, \mathrm{u} \text {,int,all within a } 3 \text {-fold }}^{\text {vio }}$ range of differences (Fig. 4C; Fig. 5C; Table 2) for both the dispersion and well-stirred models.

Alternatively, the underestimation of IVIVE for hepatic uptake clearance also could be partially explained by multifactorial mechanisms, including physiologic factors such as $\mathrm{pH}$ (Poulin and Haddad, 2015) and albumin concentration in the interstitial fluids (Poulin et al., 2016), experimental conditions (Badolo et al., 2011), and kinetic models describing the liver disposition (Iwatsubo et al., 1996). As shown in Figs. $4 \mathrm{~B}$ and $5 \mathrm{~B}$, some discrepancy remained between $f_{\mathrm{B}} \cdot C L_{\mathrm{h}, \mathrm{u}, \text { int,all }}^{\text {vivo }}$ and $f_{\mathrm{B}} \cdot P S_{\mathrm{u} \text {,inf }}^{\mathrm{vitro}}$, and the $S F$ values could be made up with various factors described above. The comprehensive review by Bowman and Benet (2018) has investigated the plausible mechanisms involved in the $S F$ value of IVIVE for hepatic uptake clearance. Determining how these factors are involved in the $S F$ values requires further investigation.

Recently, we reported an improvement in the accuracy of prediction of intrinsic hepatic clearance for three antidiabetic drugs that are substrates of both OATP1Bs and CYP2Cs by considering the "albumin-mediated" uptake in the in vitro measurements of OATP1Bmediated hepatic uptake and CYP2C-mediated metabolism (Fujino et al., 2018). Therefore, it is important to note that a kinetic model for describing the "albumin-mediated" hepatic uptake phenomenon for a drug avidly bound to albumin is useful for its robust IVIVE.

In conclusion, albumin facilitates the hepatic uptake of anionic drugs known as OATP substrates with high protein binding to hepatocytes, and the predicted $P S_{\mathrm{u} \text {,inf }}(+)$ values at 5\% HSA estimated by the "facilitated-dissociation" model provide a better extrapolation for in vivo hepatic intrinsic clearances. Furthermore, the ratios of $f_{\mathrm{B}}$. $C L_{\mathrm{h} \text {,u,int,all }}^{\text {vivo }}$ to $f_{\mathrm{B}} \cdot P S_{\mathrm{u} \text {,inf }}^{\mathrm{vitro}}$ at $5 \%$ HSA or the "albumin-mediated" factors are applicable and useful for quantifying IVIVE from the hepatic uptake clearances by isolated human hepatocytes.

\section{Authorship Contributions}

Participated in research design: Kim, Lee, Miyauchi, Sugiyama. Conducted experiments: Kim, Lee.

Contributed new reagents or analytic tools: Kim, Lee, Miyauchi, Sugiyama. Performed data analysis: Kim, Lee, Miyauchi, Sugiyama.
Wrote or contributed to the writing of the manuscript: Kim, Lee, Miyauchi, Sugiyama.

\section{References}

Badolo L, Trancart MM, Gustavsson L, and Chesné C (2011) Effect of cryopreservation on the activity of OATP1B1/3 and OCT1 in isolated human hepatocytes. Chem Biol Interact 190: $165-170$.

Blanchard N, Alexandre E, Abadie C, Lavé T, Heyd B, Mantion G, Jaeck D, Richert L, and Coassolo P (2005) Comparison of clearance predictions using primary cultures and suspensions of human hepatocytes. Xenobiotica 35:1-15.

Bounakta S, Bteich M, Mantha M, Poulin P, and Haddad S (2018) Predictions of bisphenol A hepatic clearance in the isolated perfused rat liver (IPRL): impact of albumin binding and of co-administration with naproxen. Xenobiotica 48:135-147.

Bowman CM and Benet LZ (2018) An examination of protein binding and protein-facilitated uptake relating to in vitro-in vivo extrapolation. Eur J Pharm Sci 123:502-514.

Chiba M, Ishii Y, and Sugiyama Y (2009) Prediction of hepatic clearance in human from in vitro data for successful drug development. AAPS J 11:262-276.

Colussi DM, Parisot C, Rossolino ML, Brunner LA, and Lefèvre GY (1997) Protein binding in plasma of valsartan, a new angiotensin II receptor antagonist. J Clin Pharmacol 37:214-221.

Davies B and Morris T (1993) Physiological parameters in laboratory animals and humans. Pharm Res 10:1093-1095.

Forker EL and Luxon BA (1983) Albumin-mediated transport of rose bengal by perfused rat liver. Kinetics of the reaction at the cell surface. $J$ Clin Invest 72:1764-1771.

Forker EL, Luxon BA, Snell M, and Shurmantine WO (1982) Effect of albumin binding on the hepatic transport of rose bengal: surface-mediated dissociation of limited capacity. J Pharmacol Exp Ther 223:342-347.

Fujino R, Hashizume K, Aoyama S, Maeda K, Ito K, Toshimoto K, Lee W, Ninomiya SI, and Sugiyama Y (2018) Strategies to improve the prediction accuracy of hepatic intrinsic clearance of three antidiabetic drugs: application of the extended clearance concept and consideration of the effect of albumin on CYP2C metabolism and OATP1B-mediated hepatic uptake. Eur J Pharm Sci 125:181-192.

Gulati A, Boudinot FD, and Gerk PM (2009) Binding of lopinavir to human $\alpha 1$-acid glycoprotein and serum albumin. Drug Metab Dispos 37:1572-1575.

Hatorp V (2002) Clinical pharmacokinetics and pharmacodynamics of repaglinide. Clin Pharmacokinet 41:471-483

Hirano M, Maeda K, Shitara Y, and Sugiyama Y (2004) Contribution of OATP2 (OATP1B1) and OATP8 (OATP1B3) to the hepatic uptake of pitavastatin in humans. J Pharmacol Exp Ther 311: $139-146$.

Hisaka A and Sugiyama Y (1998) Analysis of nonlinear and nonsteady state hepatic extraction with the dispersion model using the finite difference method. J Pharmacokinet Biopharm 26: 495-519.

Iwatsubo T, Hirota N, Ooie T, Suzuki H, and Sugiyama Y (1996) Prediction of in vivo drug disposition from in vitro data based on physiological pharmacokinetics. Biopharm Drug Dispos 17:273-310.

Izumi S, Nozaki Y, Komori T, Takenaka O, Maeda K, Kusuhara H, and Sugiyama Y (2017) Comparison of the predictability of human hepatic clearance for organic anion transporting polypeptide substrate drugs between different in vitro-in vivo extrapolation approaches. J Pharm Sci 106:2678-2687.

Kimoto E, Yoshida K, Balogh LM, Bi YA, Maeda K, El-Kattan A, Sugiyama Y, and Lai Y (2012) Characterization of organic anion transporting polypeptide (OATP) expression and its functional contribution to the uptake of substrates in human hepatocytes. Mol Pharm 9:3535-3542.

Miyauchi S, Masuda M, Kim SJ, Tanaka Y, Lee KR, Iwakado S, Nemoto M, Sasaki S, Shimono K, Tanaka Y, et al. (2018) The phenomenon of albumin-mediated hepatic uptake of organic anion transport polypeptide substrates: prediction of the in vivo uptake clearance from the in vitro uptake by isolated hepatocytes using a facilitated-dissociation model. Drug Metab Dispos 46: 259-267.

Miyauchi S, Sawada Y, Iga T, Hanano M, and Sugiyama Y (1993) Comparison of the hepatic uptake clearances of fifteen drugs with a wide range of membrane permeabilities in isolated rat hepatocytes and perfused rat livers. Pharm Res 10:434-440.

Pang KS and Rowland M (1977) Hepatic clearance of drugs. II. Experimental evidence for acceptance of the "well-stirred" model over the "parallel tube" model using lidocaine in the perfused rat liver in situ preparation. $J$ Pharmacokinet Biopharm 5:655-680.

Pond SM, Davis CKC, Bogoyevitch MA, Gordon RA, Weisiger RA, and Bass L (1992) Uptake of palmitate by hepatocyte suspensions: facilitation by albumin? Am J Physiol 262:G883-G894.

Poulin P, Bteich M, and Haddad S (2017) Supplemental analysis of the prediction of hepatic clearance of binary mixtures of bisphenol a and naproxen determined in an isolated perfused rat liver model to promote the understanding of potential albumin-facilitated hepatic uptake mechanism. J Pharm Sci 106:3207-3214.

Poulin P, Burczynski FJ, and Haddad S (2016) The role of extracellular binding proteins in the cellular uptake of drugs: impact on quantitative in vitro-to-in vivo extrapolations of toxicity and efficacy in physiologically based pharmacokinetic-pharmacodynamic research. J Pharm Sci 105: 497-508.

Poulin P and Haddad S (2015) Albumin and uptake of drugs in cells: additional validation exercises of a recently published equation that quantifies the albumin-facilitated uptake mechanism(s) in physiologically based pharmacokinetic and pharmacodynamic modeling research. J Pharm Sci 104:4448-4458.

Roberts MS and Rowland M (1986) A dispersion model of hepatic elimination: 1. Formulation of the model and bolus considerations. J Pharmacokinet Biopharm 14:227-260.

Seedher N and Kanojia M (2008) Reversible binding of antidiabetic drugs, repaglinide and gliclazide, with human serum albumin. Chem Biol Drug Des 72:290-296.

Shi JH, Wang Q, Pan DQ, Liu TT, and Jiang M (2017) Characterization of interactions of simvastatin, pravastatin, fluvastatin, and pitavastatin with bovine serum albumin: multiple spectroscopic and molecular docking. J Biomol Struct Dyn 35:1529-1546.

Shitara Y, Maeda K, Ikejiri K, Yoshida K, Horie T, and Sugiyama Y (2013) Clinical significance of organic anion transporting polypeptides (OATPs) in drug disposition: their roles in hepatic clearance and intestinal absorption. Biopharm Drug Dispos 34:45-78.

Tsao SC, Sugiyama Y, Sawada Y, Iga T, and Hanano M (1988a) Kinetic analysis of albumin-mediated uptake of warfarin by perfused rat liver. J Pharmacokinet Biopharm 16:165-181. 
Tsao SC, Sugiyama Y, Sawada Y, Nagase S, Iga T, and Hanano M (1986) Effect of albumin on hepatic uptake of warfarin in normal and analbuminemic mutant rats: analysis by multiple indicator dilution method. $J$ Pharmacokinet Biopharm 14:51-64.

Tsao SC, Sugiyama Y, Shinmura K, Sawada Y, Nagase S, Iga T, and Hanano M (1988b) Protein mediated hepatic uptake of rose bengal in analbuminemic mutant rats (NAR). Albumin is not indispensable to the protein-mediated transport of rose bengal. Drug Metab Dispos 16:482-489.

Watanabe T, Kusuhara H, Maeda K, Kanamaru H, Saito Y, Hu Z, and Sugiyama Y (2010) Investigation of the rate-determining process in the hepatic elimination of HMG-CoA reductase inhibitors in rats and humans. Drug Metab Dispos 38:215-222.

Watanabe T, Kusuhara H, Watanabe T, Debori Y, Maeda K, Kondo T, Nakayama H, Horita S, Ogilvie BW, Parkinson A, et al. (2011) Prediction of the overall renal tubular secretion and hepatic clearance of anionic drugs and a renal drug-drug interaction involving organic anion transporter 3 in humans by in vitro uptake experiments. Drug Metab Dispos 39:1031-1038.
Weisiger R, Gollan J, and Ockner R (1981) Receptor for albumin on the liver cell surface may mediate uptake of fatty acids and other albumin-bound substances. Science 211:1048-1051.

Weisiger RA, Zacks CM, Smith ND, and Boyer JL (1984) Effect of albumin binding on extraction of sulfobromophthalein by perfused elasmobranch liver: evidence for dissociation-limited uptake. Hepatology 4:492-501.

Address correspondence to: Dr. Yuichi Sugiyama, Sugiyama Laboratory, RIKEN Baton Zone Program, RIKEN Cluster for Science, Technology and Innovation Hub, RIKEN, 1-7-22 Suehiro-cho, Tsurumi-ku, Yokohama 230-0045, Japan. E-mail: ychi. sugiyama@riken.jp 